\title{
Intralipid for Amniotic Fluid Embolism (AFE)?
}

\author{
Joseph Eldor, Vladimir Kotlovker \\ Theoretical Medicine Institute, Jerusalem, Israel. \\ Email: csen_international@csen.com \\ Received June $29^{\text {th }}, 2012$; revised July $31^{\text {st }}, 2012$; accepted August $13^{\text {th }}, 2012$
}

\begin{abstract}
In 1998 it was first showed that intravenous Intralipid could prevent or improve resuscitation from cardiovascular collapse by severe bupivacaine overdose in rats. Since then published examples now include toxicities related to verapamil, diltiazem, amlodipine, quetiapine and sertraline, haldoperidol, lamotrigine, olanzapine, propranolol, atenolol, nevibolol, doxepin, dosulepin, imipramine, amitriptyline, glyosphate herbicide, flecainide, venlafaxine, moxidectin, and others. Amniotic fluid embolism (AFE) is a rare but potentially catastrophic obstetric emergency. Despite earlier recognition and aggressive treatment, morbidity and mortality rates remain high. An estimated $5 \%-15 \%$ of all maternal deaths in Western countries are due to AFE. The pathophysiology of AFE is not completely understood. AFE most commonly occurs during labor, delivery, or the immediate postpartum period. However, it has been reported to occur up to $48 \mathrm{~h}$ postpartum. Pulmonary hypertension and right heart strain/failure may be the result of physical amniotic fluid debris in the pulmonary vasculature or, perhaps more likely, result from circulating pulmonary vasoconstrictive mediators. Therapy with Intralipid in male rats resulted in $100 \%$ survival and prevented Pulmonary arterial hypertension-induced right ventricular failure by preserving right ventricular pressure and right ventricular ejection fraction and preventing right ventricular hypertrophy and lung remodeling. In preexisting severe Pulmonary arterial hypertension, Intralipid attenuated most lung and right ventricular abnormalities. The beneficial effects of Intralipid in Pulmonary arterial hypertension seem to result from the interplay of various factors, among which preservation and/or stimulation of angiogenesis, suppression and/or reversal of inflammation, fibrosis and hypertrophy, in both lung and right ventricular, appear to be major contributors. In conclusion, Intralipid not only prevents the development of Pulmonary arterial hypertension and right ventricular failure but also rescues preexisting severe Pulmonary arterial hypertension. Intralipid treatment is a new treatment for AFE (amniotic fluid embolism) which was never suggested before. Animal studies should be done in order to evaluate this new treatment modality.
\end{abstract}

Keywords: Intralipid; Bupivacaine Toxicity; Amniotic Fluid Embolism

\section{Introduction}

Weinberg et al. [1] first showed in 1998 that an infusion of a soybean oil emulsion normally used as a total parenteral nutrition solution could prevent (by pretreatment) or improve resuscitation from cardiovascular collapse caused by severe bupivacaine overdose in the intact, anesthetized rat. Subsequent studies from the same laboratory confirmed these findings in isolated rat heart [2] and anesthetized $\operatorname{dog}[3]$.

Under the latter experimental model, return of spontaneous circulation after a bupivacaine challenge occurred in all animals receiving lipid, but in none of the saline controls [3]. This study was accompanied by an editorial asking whether lipid might be the long-sought "silver bullet" for local anesthetic systemic toxicity (LAST). Since then, the effectiveness of lipid emulsion infusion in reversing LAST has been confirmed in other laboratories and by systematic analysis [4] in the clinical setting as well.

These publications, along with other animal studies, opened the door to more widespread use of lipid emulsion for emergency treatment of toxicities caused by a range of lipophilic drugs. Notably, published examples now include toxicities related to verapamil, diltiazem, amlodipine, quetiapine and sertraline, haldoperidol, lamotrigine, olanzapine, propranolol, atenolol, nevibolol, doxepin, dosulepin, imipramine, amitriptyline, glyosphate herbicide, flecainide, venlafaxine, moxidectin, and others.

\section{Material and Method}

Tricyclic antidepressant (TCA) toxicity results predominantly from myocardial sodium-channel blockade. Subsequent ventricular dysrhythmias, myocardial depression, and hypotension cause cardiovascular collapse. Animal studies have demonstrated the effectiveness of intravenous lipid-emulsion in treating TCA cardiotoxicity. 
Blaber M. S. et al. [5] report a case of dothiepin (tricyclic antidepressant) overdose causing refractory cardiovascular collapse, which seemed to be successfully reversed with lipid-emulsion therapy $\left(\right.$ Intralipid $^{\circledR}$; Fresenius, Cheshire, UK).

Lipid emulsions are a potentially novel therapy for reversing cardiotoxicity seen in TCA overdose. Research is required into the role of lipid emulsion in the management of poisoning by oral lipophilic agents.

Lipid infusion is useful in reversing cardiac toxicity of local anesthetics, and recent reports indicate it may be useful in resuscitation from toxicity induced by a variety of other drugs. While the mechanism behind the utility of lipid rescue remains to be fully elucidated, the predominant effect appears to be creation of a "lipid sink".

French D. et al. [6] tried to determine whether the extraction of drugs by lipid, and hence the clinical efficacy of lipid rescue in toxicological emergencies can be predicted by specific drug properties.

Each drug investigated was added individually to human drug-free serum. Intralipid ${ }^{\mathbb{R}}$ was added to this drugcontaining serum, shaken and then incubated at $37^{\circ} \mathrm{C}$. The lipid was removed by ultracentrifugation and the concentration of drug remaining in the serum was measured by high-pressure liquid chromatography.

In this in vitro model, the ability of lipid emulsion to bind a drug was largely dependent upon the drug's lipid partition constant. Additionally, using a multiple linear regression model, the prediction of binding could be improved by combining the lipid partition constant with the volume of distribution together accounting for approximately $88 \%$ of the variation in the decrease in serum drug concentration with the administration of lipid emulsion.

The lipid partition constant and volume of distribution can likely be used to predict the efficacy of lipid infusion in reversing the cardiac toxicity induced by anesthetics or other medications.

Local anaesthetics may induce cardiac arrest, usually because of rapid absorption from the site of injection or because of an intended intravascular injection. Early central nervous system symptoms usually precede seizures. Cardiac arrhythmias follow the CNS signs. These arrhythmias often resolve with the i.v. bolus injection of 100 to $150 \mathrm{~mL}$ of a lipid emulsion $\left(20 \%\right.$ Intralipid $\left.^{\circledR}\right)$. Although long acting local anaesthetics (bupivacaine, ropivacaine, levobupivacaine) are predominantly involved in this cardiac toxicity, lidocaine may also induce cardiac arrhythmias and clinician must be aware of this risk. In case of cardiac arrest, resuscitation manoeuvres are of major importance. They need to be performed immediately and the efficacy of the lipid rescue requires a correct coronary flow to be efficacious. Finally, prevention is the key of a safe injection. It is important to control the dose, to inject slowly, without any excessive pressure and to verify that no blood reflux occurs [7].

Papadopoulou A. et al. [8] hypothesized that by substituting a dye surrogate in place of local anesthetic, they could visually demonstrate dye sequestration by lipid emulsion that would be dependent on both dye lipophilicity and the amount of lipid emulsion used.

They selected 2 lipophilic dyes, acid blue 25 and victoria blue, with $\log \mathrm{P}$ values comparable to lidocaine and bupivacaine, respectively. Each dye solution was mixed with combinations of lipid emulsion and water to emulate "lipid rescue" treatment at dye concentrations equivalent to fatal, cardiotoxic, and neurotoxic local anesthetic plasma concentrations. The lipid emulsion volumes added to each dye solution emulated equivalent intravenous doses of 100,500 , and $900 \mathrm{~mL}$ of $20 \%$ Intralipid in a 75-kg adult. After mixing, the samples were separated into a lipid-rich supernatant and a lipid-poor subnatant by heparin flocculation. The subnatants were isolated, and their colors compared against a graduated dye concentration scale.

Lipid emulsion addition resulted in significant dye acquisition by the lipid compartment accompanied by a reduction in the color intensity of the aqueous phase that could be readily observed. The greatest amount of sequestration occurred with the dye possessing the higher $\log \mathrm{P}$ value and the greatest amount of lipid emulsion.

This study provides a visual demonstration of the lipid sink effect. It supports the theory that lipid emulsion may reduce the amount of free drug present in plasma from concentrations associated with an invariably fatal outcome to those that are potentially survivable.

\section{Statistical Analysis}

Local anesthetic (LA) intoxication with cardiovascular arrest is a potential fatal complication of regional anesthesia. Lipid resuscitation has been recommended for the treatment of LA-induced cardiac arrest. Aim of the study [9] was to compare four different rescue regimens using epinephrine and/or lipid emulsion and vasopressin to treat cardiac arrest caused by bupivacaine intoxication.

Twenty-eight piglets were randomized into four groups $(4 \times 7)$, anesthetized with sevoflurane, intubated, and ventilated. Bupivacaine was infused with a syringe driver via central venous catheter at a rate of $1 \mathrm{mg} \cdot \mathrm{kg}^{-1} \cdot \mathrm{min}^{-1}$ until circulatory arrest. Bupivacaine infusion and sevoflurane were then stopped, chest compression was started, and the pigs were ventilated with $100 \%$ oxygen. After 1 min, epinephrine $10 \mu \mathrm{g} \cdot \mathrm{kg}^{-1}$ (group 1), Intralipid ${ }^{\mathbb{B}} 20 \% 4$ $\mathrm{ml} \cdot \mathrm{kg}^{-1}$ (group 2), epinephrine $10 \mu \mathrm{g} \cdot \mathrm{kg}^{-1}+$ Intralipid $^{\circledR} 4$ $\mathrm{ml} \cdot \mathrm{kg}^{-1}$ (group 3) or 2 IU vasopressin + Intralipid $^{\circledR} 4$ $\mathrm{ml} \cdot \mathrm{kg}^{-1}$ (group 4) were administered. Secondary epinephrine doses were given after $5 \mathrm{~min}$ if required. 
Survival was $71 \%, 29 \%, 86 \%$, and $57 \%$ in groups 1,2 , 3 , and 4 . Return of spontaneous circulation was regained only by initial administration of epinephrine alone or in combination with Intralipid ${ }^{\circledR}$. Piglets receiving the combination therapy survived without further epinephrine support. In contrast, in groups 2 and 4, return of spontaneous circulation was only achieved after secondary epinephrine rescue.

In cardiac arrest caused by bupivacaine intoxication, first-line rescue with epinephrine and epinephrine + Intralipid $^{\circledR}$ was more effective with regard to survival than Intralipid $^{\circledR}$ alone and vasopressin + Intralipid $^{\circledR}$ in this pig model [9].

Local anesthetic (LA) intoxication with severe hemodynamic compromise is a potential catastrophic event. Lipid resuscitation has been recommended for the treatment of LA-induced cardiac arrest. However, there are no data about effectiveness of Intralipid for the treatment of severe cardiovascular compromise prior to cardiac arrest. Aim of this study was to compare effectiveness of epinephrine and Intralipid for the treatment of severe hemodynamic. Piglets were compromise owing to bupivacaine intoxication, anesthetized with sevoflurane, intubated, and ventilated. Bupivacaine was infused with a syringe driver via a central venous catheter at a rate of 1 $\mathrm{mg} \cdot \mathrm{kg}^{-1} \cdot \mathrm{min}^{-1}$ until invasively measured mean arterial pressure (MAP) dropped to $50 \%$ of the initial value. Bupivacaine infusion was then stopped, and epinephrine 3 $\mu \mathrm{g} \cdot \mathrm{kg}^{-1}$ (group 1), Intralipid ${ }^{\mathbb{B}} 20 \% 2 \mathrm{ml} \cdot \mathrm{kg}^{-1}$ (group 2), or Intralipid $20 \% 4 \mathrm{ml} \cdot \mathrm{kg}^{-1}$ (group 3) was immediately administered. Twenty-one piglets $(3 \times 7)$, were recorded. All animals in group $1(100 \%)$ but only four of seven (57\%) piglets in groups 2 and 3, respectively, survived. Normalization of hemodynamic parameters (HR, MAP) and ET $\left(\mathrm{CO}_{2}\right)$ was fastest in group 1 with all piglets achieving HR and MAP values. hemodynamic compromise owing to bupivacaine intoxication in piglets, firstline rescue with epinephrine was more effective than Intralipid with regard to survival as well as normalization of hemodynamic parameters and ET $\left(\mathrm{CO}_{2}\right)$ [10].

Intravenous lipid emulsion (ILE) has been proposed as a rescue therapy for severe local anesthetic drugs toxicity, but experience is limited with other lipophilic drugs. An 18 -year-old healthy woman was admitted $8 \mathrm{~h}$ after the voluntary ingestion of sustained-release diltiazem (3600 $\mathrm{mg}$ ), with severe hypotension refractory to fluid therapy, calcium salts, and high-dose norepinephrine $(6.66 \mu \mathrm{g} / \mathrm{kg} /$ $\mathrm{min}$ ). Hyperinsulinemic euglycemia therapy was initiated and shortly after was followed by a protocol of ILE (intralipid $20 \%, 1.5 \mathrm{ml} / \mathrm{kg}$ as bolus, followed by $0.25 \mathrm{ml} / \mathrm{kg}$ over $1 \mathrm{~h}$ ). The main finding attributed to ILE was an apparent rapid decrease in insulin resistance, despite a prolonged serum diltiazem elimination half-life. Diltiazem is a lipophilic cardiotoxic drug, which could be sequestered in an expanded plasma lipid phase. The mechanism of action of ILE is not known, including its role in insulin resistance and myocardial metabolism in calcium-channel blocker poisoning [11].

There is increasing evidence for the use of Intralipid in the management of acute local anaesthetic toxicity. This is supported by the recent Association of Anaesthetists of Great Britain and Ireland (AAGBI) guidelines for the management of local anaesthetic toxicity. Acute hospitals in England and Wales were surveyed to determine the proportion that currently stocked Intralipid, the locations of stocks within the hospital, guidelines related to its use and previous use in the last 12 months. The majority of hospitals surveyed stocked Intralipid in multiple locations, although not in all areas using high volumes of local anaesthetics. Guidelines were typically in place, although these were often local rather than those from the AAGBI. Use in the last 12 months was uncommon, but typically information was not available on indications for its use. More systematic data collection is required on the safety and efficacy of Intralipid in the management of acute drug toxicity [12].

Intralipid therapy has been used successfully as "rescue therapy" in several cases of overdose. West P. L. et $a l$. [13] present a case of iatrogenic lipid emulsion overdose because of a dosing error.

A 71-year-old female overdosed on 27 tablets of $5 \mathrm{mg}$ amlodipine. Although initially stable in the Emergency Department, she became hypotensive, oliguric, and respiratory failure developed despite medical therapy. The primary treating team felt that meaningful recovery was unlikely to occur without rapid improvement in clinical status, and $12.5 \mathrm{~h}$ after presentation, Intralipid rescue therapy was initiated. A protocol for Intralipid specifying a maximum infusion of $400 \mathrm{~mL}$ of $20 \%$ lipid emulsion was faxed, but the infusion was continued until $2 \mathrm{~L}$ of lipid emulsion was infused. There were no detectable adverse hemodynamic effects of the Intralipid infusion. After this time, laboratory values were difficult to obtain. Three hours after the infusion, a metabolic panel was obtained from ultracentrifuged blood showing hyponatremia. A white blood cell (WBC) was obtained from a complete blood count (CBC) performed $22 \mathrm{~h}$ after the infusion, hemoglobin and hematocrit could not be obtained from this blood. A platelet count was obtained by smear estimate. Hematocrits were obtained from centrifuged blood and appeared elevated. No oxygenation could be obtained on blood gas. The patient's family chose to withdraw care on hospital day 2 and no further laboratory draws were obtained. Amlodipine was $1500 \mathrm{ng} / \mathrm{mL}$ (ref. $3-11 \mathrm{ng} / \mathrm{mL}$ ).

Lipid emulsion overdose caused no detectable acute adverse hemodynamic effects. The following laboratory values were unobtainable immediately after infusion: 
white blood cell count, hemoglobin, hematocrit, platelet count, and a metabolic panel of serum electrolytes. Ultracentrifugation of blood allowed for detection of a metabolic panel $3 \mathrm{~h}$ after the infusion. Centrifuged hematocrits appeared to be higher than expected.

Lipid infusion reverses systemic local anesthetic toxicity. The acceptable upper limit for lipid administration is unknown and has direct bearing on clinical management. Hiller D. B. et al. [14] hypothesize that high volumes of lipid could have undesirable effects and sought to identify the dose required to kill $50 \%$ of the animals $(\operatorname{LD}(50))$ of large volume lipid administration.

Intravenous lines and electrocardiogram electrodes were placed in anesthetized, male Sprague-Dawley rats. Twenty percent lipid emulsion $(20,40,60$, or $80 \mathrm{~mL} / \mathrm{kg}$ ) or saline $(60$ or $80 \mathrm{~mL} / \mathrm{kg}$ ), were administered over 30 mins; lipid dosing was assigned by the Dixon "up-anddown" method. Rats were recovered and observed for 48 $\mathrm{h}$ then euthanized for histologic analysis of major organs. Three additional rats were administered $60 \mathrm{~mL} / \mathrm{kg}$ lipid emulsion and euthanized at 1,4 , and $24 \mathrm{~h}$ to identify progression of organ damage.

The maximum likelihood estimate for $\operatorname{LD}(50)$ was 67.72 (SE, 10.69) mL/kg. Triglycerides were elevated immediately after infusion but returned to baseline by 48 $\mathrm{h}$ when laboratory abnormalities included elevated amylase, aspartate aminotransferase, and serum urea nitrogen for all lipid doses. Histologic diagnosis of myocardium, brain, pancreas, and kidneys was normal at all doses. Microscopic abnormalities in lung and liver were observed at 60 and $80 \mathrm{~mL} / \mathrm{kg}$; histopathology in the lung and liver was worse at $1 \mathrm{~h}$ than at 4 and $24 \mathrm{~h}$.

The $\mathrm{LD}(50)$ of rapid, high volume lipid infusion is an order of magnitude greater than doses typically used for lipid rescue in humans and supports the safety of lipid infusion at currently recommended doses for toxin-induced cardiac arrest. Lung and liver histopathology was observed at the highest infused volumes.

\section{Results}

Cave G. and Harvey M. [15] evaluate the efficacy of lipid emulsion as antidotal therapy outside the accepted setting of local anesthetic toxicity.

Literature was accessed through PubMed, OVID (1966February 2009), and EMBASE (1947-February 2009) using the search terms "intravenous" and ["fat emulsion" or "lipid emulsion" or "Intralipid"] and ["toxicity" or "resuscitation" or "rescue" or "arrest" or "antidote"]. Additional author and conference publication searches were undertaken. Publications describing the use of lipid emulsion as antidotal treatment in animals or humans were included.

Fourteen animal studies, one human study, and four case reports were identified. In animal models, intrave- nous lipid emulsion (ILE) has resulted in amelioration of toxicity associated with cyclic antidepressants, verapamil, propranolol, and thiopentone. Administration in human cases has resulted in successful resuscitation from combined bupropion/lamotrigine-induced cardiac arrest, reversal of sertraline/quetiapine-induced coma, and amelioration of verapamil- and beta blocker-induced shock.

Management of overdose with highly lipophilic cardiotoxic medications should proceed in accord with established antidotal guidelines and early poisons center consultation. Data from animal experiments and human cases are limited, but suggestive that ILE may be helpful in potentially lethal cardiotoxicity or developed cardiac arrest attributable to such agents. Use of lipid emulsion as antidote remains a nascent field warranting further preclinical study and systematic reporting of human cases of use.

Previous investigators have demonstrated amelioration of lipid-soluble drug toxidromes with infusion of lipid emulsions. Clomipramine is a lipid-soluble tricyclic antidepressant with significant cardiovascular depressant activity in human overdose. Harvey M. and Cave G. [16] compare resuscitation with Intralipid versus sodium bicarbonate in a rabbit model of clomipramine toxicity.

Thirty sedated and mechanically ventilated New Zealand White rabbits were infused with clomipramine at $320 \mathrm{mg} / \mathrm{kg}$ per hour. At target mean arterial pressure of $50 \%$ initial mean arterial pressure, animals were rescued with $0.9 \% \mathrm{NaCl} 12 \mathrm{~mL} / \mathrm{kg}, 8.4 \%$ sodium bicarbonate 3 $\mathrm{mL} / \mathrm{kg}$, or $20 \%$ Intralipid $12 \mathrm{~mL} / \mathrm{kg}$. Pulse rate, mean arterial pressure, and QRS duration were sampled at 2.5minute intervals to 15 minutes. In the second phase of the experiment, 8 sedated and mechanically ventilated rabbits were infused with clomipramine at $240 \mathrm{mg} / \mathrm{kg}$ per hour to a mean arterial pressure of $25 \mathrm{~mm} \mathrm{Hg}$. Animals received either $2 \mathrm{~mL} / \mathrm{kg} 8.4 \%$ sodium bicarbonate or 8 $\mathrm{mL} / \mathrm{kg} \mathrm{20 \%}$ Intralipid as rescue therapy. External cardiac compression and intravenous adrenaline were administered in the event of cardiovascular collapse.

Mean difference in mean arterial pressure between Intralipid- and saline solution-treated groups was $21.1 \mathrm{~mm}$ $\mathrm{Hg}(95 \%$ confidence interval [CI] 13.5 to $28.7 \mathrm{~mm} \mathrm{Hg})$ and $19.5 \mathrm{~mm} \mathrm{Hg}(95 \% \mathrm{CI} 10.5$ to $28.9 \mathrm{~mm} \mathrm{Hg})$ at 5 and 15 minutes, respectively. Mean difference in mean arterial pressure between Intralipid- and bicarbonate-treated groups was $19.4 \mathrm{~mm} \mathrm{Hg}$ (95\% CI 18.8 to $27.0 \mathrm{~mm} \mathrm{Hg}$ ) and $11.5 \mathrm{~mm} \mathrm{Hg}(95 \%$ CI 2.5 to $20.5 \mathrm{~mm} \mathrm{Hg})$ at 5 and 15 minutes. The rate of change in mean arterial pressure was greatest in the Intralipid-treated group at 3 minutes (6.2 $\mathrm{mm} \mathrm{Hg} / \mathrm{min}$ [95\% CI 3.8 to $8.6 \mathrm{~mm} \mathrm{Hg} / \mathrm{min}$ ]. In the second phase of the experiment spontaneous circulation was maintained in all Intralipid-treated rabbits $(n=4)$. All animals in the bicarbonate-treated group developed pulseless electrical activity and proved refractory to re- 
suscitation at 10 minutes $(\mathrm{n}=4, \mathrm{P}=0.023)$.

In this rabbit model, Intralipid infusion resulted in more rapid and complete reversal of clomipramine-induced hypotension compared with sodium bicarbonate. Additionally, Intralipid infusion prevented cardiovascular collapse in a model of severe clomipramine toxicity.

\section{Discussion}

Amniotic fluid embolism (AFE) is a rare but potentially catastrophic obstetric emergency. Despite earlier recognition and aggressive treatment, morbidity and mortality rates remain high. An estimated 5\% - 15\% of all maternal deaths in Western countries are due to AFE [17].

Recent retrospective reviews of population-based hospital databases in Canada [18] and the United States [19] found AFE incidences of 6.1 - 7.7 cases per 100,000 births.

Early studies revealed mortality rates as high as $61 \%$ $86 \%$, but more recent estimates suggest a case fatality of $13 \%-26 \%$ [19-21].

First reported by Meyer in 1926 [22], and then later identified as a syndrome in 1941 by Steiner and Lushbaugh [23].

The pathophysiology of AFE is not completely understood. AFE most commonly occurs during labor, delivery, or the immediate postpartum period. However, it has been reported to occur up to $48 \mathrm{~h}$ postpartum [24]. Once thought to be the result of an actual embolic obstruction of the pulmonary vasculature by components of amniotic fluid, AFE might result from immune activation and present as an anaphylactoid process. AFE likely involves a spectrum of severity from a subclinical process to a catastrophic event. Early recognition and prompt and aggressive resuscitative efforts enhance the probability of maternal and neonatal survival.

Three phases in the clinical course of AFE have been described. The first or immediate phase is often characterized by altered mental status, respiratory distress, peripheral oxygen desaturation, and hemodynamic collapse. The second phase involves coagulopathy and hemorrhage and occurs in an estimated $4 \%-50 \%$ of patients with presumed AFE. Although older studies of AFE required either sudden, unresuscitatable maternal death or the subsequent development of disseminated intravascular coagulation (DIC) for inclusion in the AFE database, it is now recognized that DIC does not develop in all cases of AFE. Tissue injury and end-organ system failure comprise the last phase of AFE. Clinical findings will vary depending on the organ system(s) predominantly affected. Ventilation-perfusion mismatching as a result of pulmonary vascular constriction at the onset of AFE may explain sudden hypoxia and respiratory arrest [25].

Pulmonary hypertension and right-heart strain/failure may be the result of physical amniotic fluid debris in the pulmonary vasculature or, perhaps more likely, result from circulating pulmonary vasoconstrictive mediators. The mechanisms for myocardial dysfunction that lead to early hypotension are multifactorial. Proposed explanations include myocardial failure in response to sudden pulmonary hypertension, a direct myocardial depressant effect of humoral mediators in amniotic fluid, deviation of the intraventricular septum due to right ventricular dilation, and/or ischemic myocardial injury from hypoxemia [26-28].

Pulmonary arterial hypertension (PAH) is characterized by pulmonary vascular remodeling leading to right ventricular (RV) hypertrophy and failure. Intralipid (ILP), a source of parenteral nutrition for patients, contains $\gamma$ linolenic acid and soy-derived phytoestrogens that are protective for lungs and heart. Umar S. et al. [29] investigated the therapeutic potential of ILP in preventing and rescuing monocrotaline-induced $\mathrm{PAH}$ and RV dysfunction. PAH was induced in male rats with monocrotaline $(60 \mathrm{mg} / \mathrm{kg})$. Rats then received daily ILP (1 mL of $20 \%$ ILP per day IP) from day 1 to day 30 for prevention protocol or from day 21 to day 30 for rescue protocol. Other monocrotaline-injected rats were left untreated to develop severe PAH by day 21 or RV failure by approximately day 30. Saline or ILP-treated rats served as controls. Significant increase in RV pressure and decrease in $\mathrm{RV}$ ejection fraction in the RV failure group resulted in high mortality. Therapy with ILP resulted in 100\% survival and prevented PAH-induced RV failure by preserving RV pressure and RV ejection fraction and preventing RV hypertrophy and lung remodeling. In preexisting severe PAH, ILP attenuated most lung and RV abnormalities. The beneficial effects of ILP in PAH seem to result from the interplay of various factors, among which preservation and/or stimulation of angiogenesis, suppression and/or reversal of inflammation, fibrosis and hypertrophy, in both lung and RV, appear to be major contributors. In conclusion, ILP not only prevents the development of PAH and RV failure but also rescues preexisting severe PAH [29].

\section{Conclusion}

Intralipid treatment is a new treatment for AFE which was never suggested before. Animal studies should be done in order to evaluate this new treatment modality.

\section{REFERENCES}

[1] G. L. Weinberg, T. VadeBoncouer, G. A. Ramaraju, M. F. Garcia-Amaro and M. J. Cwik, "Pretreatment or Resuscitation with a Lipid Infusion Shifts the Dose-Response to Bupivacaine-Induced Asystole in Rats," Anesthesiology, Vol. 88, No. 4, 1998, pp. 1071-1075. doi:10.1097/00000542-199804000-00028 
[2] G. L. Weinberg, R. Ripper, P. Murphy, L. B. Edelman, W. Hoffman, G. Strichartz and D. L. Feinstein, "Lipid Infusion Accelerates Removal of Bupivacaine and Recovery from Bupivacaine Toxicity in the Isolated Rat Heart," Regional Anesthesia and Pain Medicine, Vol. 31, No. 4, 2006, pp. 296-303.

[3] G. Weinberg, R. Ripper, D. L. Feinstein and W. Hoffman, "Lipid Emulsion Infusion Rescues Dogs from Bupivacaine-Induced Cardiac Toxicity," Regional Anesthesia and Pain Medicine, Vol. 28, No. 3, 2003, pp. 198-202.

[4] C. Jamaty, B. Bailey, A. Larocque, E. Notebaert, K. Sanogo and J. M. Chauny, "Lipid Emulsions in the Treatment of Acute Poisoning: A Systematic Review of Human and Animal Studies," Clinical Toxicology, Vol. 48, No. 1, 2010, pp. 1-27

[5] M. S. Blaber, J. N. Khan, J. A. Brebner and R. McColm, "Lipid Rescue for Tricyclic Antidepressant Cardiotoxicity," The Journal of Emergency Medicine, 2012, in press. doi:10.1016/j.jemermed.2011.09.010

[6] D. French, C. Smollin, W. Ruan, A. Wong, K. Drasner and A. H. Wu, "Partition Constant and Volume of Distribution as Predictors of Clinical Efficacy of Lipid Rescue for Toxicological Emergencies," Clinical Toxicology, Vol. 49, No. 9, 2011, pp. 801-809. doi:10.3109/15563650.2011.617308

[7] J. X. Mazoit, "Cardiac Arrest and Local Anaesthetics," La Presse Médicale, 2012, in press. doi:10.1016/j.lpm.2012.04.016

[8] A. Papadopoulou, J. W. Willers, T. L. Samuels and D. R. Uncles, "The Use of Dye Surrogates to Illustrate Local Anesthetic Drug Sequestration by Lipid Emulsion: A Visual Demonstration of the Lipid Sink Effect," Regional Anesthesia and Pain Medicine, Vol. 37, No. 2, 2012, pp. 183-187.

[9] J. Mauch, O. M. Jurado, N. Spielmann, R. Bettschart-Wolfensberger and M. Weiss, "Resuscitation Strategies from Bupivacaine-Induced Cardiac Arrest," Pediatric Anesthesia, Vol. 22, No. 2, 2012, pp. 124-129. doi:10.1111/j.1460-9592.2011.03688.x

[10] J. Mauch, O. M. Jurado, N. Spielmann, R. Bettschart-Wolfensberger and M. Weiss, "Comparison of Epinephrine vs Lipid Rescue to Treat Severe Local Anesthetic ToxicityAn Experimental Study in Piglets," Pediatric Anesthesia, Vol. 21, No. 11, 2011, pp. 1103-1108. doi:10.1111/j.1460-9592.2011.03652.x

[11] V. Montiel, T. Gougnard and P. Hantson, "Diltiazem Poisoning Treated with Hyperinsulinemic Euglycemia Therapy and Intravenous Lipid Emulsion," European Journal of Emergency Medicine, Vol. 18, No. 2, 2011, pp. 121-123. doi:10.1097/MEJ.0b013e32834130ab

[12] P. Hamann, P. I. Dargan, N. Parbat, H. Ovaska and D. M. Wood, "Availability of and Use of Intralipid (Lipid Rescue Therapy, Lipid Emulsion) in England and Wales," The Journal of Emergency Medicine, Vol. 27, No. 8, 2010, pp. 590-592. doi:10.1136/emj.2009.083352

[13] P. L. West, N. J. McKeown and R. G. Hendrickson, "Iatrogenic Lipid Emulsion Overdose in a Case of Amlodipine Poisoning," Clinical Toxicology, Vol. 48, No. 4, 2010, pp. 393-396. doi:10.3109/15563651003670843
[14] D. B. Hiller, G. D. Gregorio, K. Kelly, R. Ripper, L. Edelman, R. Boumendjel, K. Drasner and G. L. Weinberg, "Safety of High Volume Lipid Emulsion Infusion: A First Approximation of LD50 in Rats," Regional Anesthesia and Pain Medicine, Vol. 35, No. 2, 2010, pp. 140-144. doi:10.1097/AAP.0b013e3181c6f5aa

[15] G. Cave and M. Harvey, "Intravenous Lipid Emulsion as Antidote beyond Local Anesthetic Toxicity: A Systematic Review," Academic Emergency Medicine, Vol. 16, No. 9, 2009, pp. 815-824. doi:10.1111/j.1553-2712.2009.00499.x

[16] M. Harvey and G. Cave, "Intralipid Outperforms Sodium Bicarbonate in a Rabbit Model of Clomipramine Toxicity," Annals of Emergency Medicine, Vol. 49, No. 2, 2007, pp. 178-185doi:10.1016/j.annemergmed.2006.07.016

[17] A. Conde-Agudelo and R. Romero, "Amniotic Fluid Embolism: An Evidence-Based Review," American Journal of Obstetrics \& Gynecology, Vol. 201, No. 5, 2009, p. 445. doi:10.1016/j.ajog.2009.04.052

[18] M. S. Kramer, J. Rouleau, T. F. Baskett and K. S. Joseph, "Maternal Health Study Group of the Canadian Perinatal Surveillance System: Amniotic-Fluid Embolism and Medical Induction of Labour: A Retrospective, PopulationBased Cohort Study," Lancet, Vol. 368, No. 9545, 2006, pp. 1444-1448doi:10.1016/S0140-6736(06)69607-4

[19] H. A. Abenhaim, L. Azoulay, M. S. Kramer and L. Leduc, "Incidence and Risk Factors of Amniotic Fluid Embolisms: A Population-Based Study on 3 Million Births in the United States," American Journal of Obstetrics \& Gynecology, Vol. 199, No. 1, 2008, p. 49. doi:10.1016/j.ajog.2007.11.061

[20] M. Knight, D. Tuffnell, P. Brocklehurst, P. Spark and J. J. Kurinczuk, "UK Obstetric Surveillance System: Incidence and Risk Factors for Amniotic-Fluid Embolism," Obstetrics \& Gynecology, Vol. 115, No. 5, 2010, pp. 910-917. doi:10.1097/AOG.0b013e3181d9f629

[21] A. F. Gei, R. B. Vadhera and G. D Hankins, "Embolism during Pregnancy: Thrombus, Air, and Amniotic Fluid," Anesthesiology Clinics of North America, Vol. 21, No. 1, 2003, pp. 165-182. doi:10.1016/S0889-8537(02)00052-4

[22] J. R. Meyer, "Embolia Pulmonar Amnio Caseosa," Brasil Medico, Vol. 2, 1926, pp. 301-303.

[23] P. E. Steiner and C. C. Lushbaugh, "Maternal Pulmonary Embolism by Amniotic Fluid as a Cause of Obstetric Shock and Unexpected Deaths in Obstetrics," Journal of the American Medical Association, Vol. 255, No. 16, 1986, pp. 2187-2203. doi:10.1001/jama.1986.03370160085032

[24] A. Conde-Agudelo and R. Romero, "Amniotic Fluid Embolism: An Evidence-Based Review," American Journal of Obstetrics \& Gynecology, Vol. 201, No. 5, 2009, p. 445. doi:10.1016/j.ajog.2009.04.052

[25] S. L. Clark, G. D. Hankins, D. A. Dudley, G. A. Dildy and T. F. Porter, "Amniotic Fluid Embolism: Analysis of the National Registry," American Journal of Obstetrics \& Gynecology, Vol. 172, No. 4, 1995, pp. 1158-1167.

[26] A. O'Shea and S. Eappen, "Amniotic Fluid Embolism," International Anesthesiology Clinics, Vol. 45, No. 1, 2007, pp. 17-28. doi:10.1097/AIA.0b013e31802b8853 
[27] R. D. Stanten, L. I. Iverson, T. M. Daugharty, S. M. Lovett, C. Terry and E. Blumenstock, "Amniotic Fluid Embolism Causing Catastrophic Pulmonary Vasoconstriction: Diagnosis by Transesophageal Echocardiogram and Treatment by Cardiopulmonary Bypass," Obstetrics \& Gynecology, Vol. 102, No. 3, 2003, pp. 496-498. doi:10.1016/S0029-7844(03)00161-3

[28] C. F. James, N. G. Feinglass, D. M. Menke, S. F. Grinton and T. J. Papadimos, "Massive Amniotic Fluid Embolism: Diagnosis Aided by Emergency Transesophageal Echo- cardiography," International Journal of Obstetric Anesthesia, Vol. 13, No. 4, 2004, pp. 279-283.

doi:10.1016/j.ijoa.2004.03.008

[29] S. Umar, R. D. Nadadur, J. Li, F. Maltese, P. Partownavid, A. van der Laarse and M. Eghbali, "Intralipid Prevents and Rescues Fatal Pulmonary Arterial Hypertension and Right Ventricular Failure in Rats," Hypertension, Vol. 58, No. 3, 2011, pp. 512-518. doi:10.1161/HYPERTENSIONAHA.110.168781 